\title{
Association between synovial fluid levels of aggrecan ARGS fragments and radiographic progression in knee osteoarthritis
}

\author{
Staffan Larsson ${ }^{1 *}$, Martin Englund ${ }^{1,2}$, André Struglics $^{1}$, L Stefan Lohmander ${ }^{1}$
}

\begin{abstract}
Introduction: Aggrecanase cleavage at the ${ }^{392} \mathrm{Glu}^{-393} \mathrm{Ala}$ bond in the interglobular domain (IGD) of aggrecan, releasing $\mathrm{N}$-terminal ${ }^{393} \mathrm{ARGS}$ fragments, is an early key event in arthritis and joint injuries. We determined whether synovial fluid (SF) levels of ARGS-aggrecan distinguish subjects with progressive radiographic knee osteoarthritis (ROA) from those with stable or no ROA.

Methods: We studied 141 subjects who, at examination A, had been given meniscectomies an average of 18 years earlier (range, 15 to 22 years). Seventeen individuals without surgery, and without known injury to the menisci or cruciate ligaments, were used as references. At examinations A and B, with a mean follow-up time of 7.5 years, we obtained SF and standing tibiofemoral and skyline patellofemoral radiographs. SF ARGS-aggrecan was measured with an electrochemiluminescence immunoassay, and we graded radiographs according to the OARSI atlas. The association between SF ARGS levels at examination A and progression of radiographic features of knee OA between examinations $A$ and $B$ was assessed by using logistic regression adjusted for age, gender, body mass index, and time between examinations, and stratified by ROA status at examination A.

Results: We found a weak negative association between SF ARGS concentrations and loss of joint space: the likelihood of progression of radiographic joint space narrowing decreased 0.9 times per picomole per milliliter increase in ARGS (odds ratio (OR) 0.89; $95 \%$ confidence interval (CI), 0.79 to 0.996). In subjects with and without preexisting ROA at examination $\mathrm{A}$, the association was $\mathrm{OR}, 0.96$; 0.81 to 1.13 ; and 0.77 ; 0.62 to 0.95 , respectively. Average levels of SF ARGS 18 years after meniscectomy were no different from those of reference subjects and were not correlated to radiographic status at examination $\mathrm{A}$.
\end{abstract}

Conclusions: In subjects with previous knee meniscectomy but without ROA, levels of SF ARGS-aggrecan were weakly and inversely associated with increased loss of joint space over a period of 7.5 years.

\section{Introduction}

In osteoarthritis (OA), the balance between cartilagematrix synthesis and degradation is disturbed, resulting in a gradual destruction of the articular cartilage [1]. Collagen type II and aggrecan are the two major constituents of the matrix, and their proteolysis is regarded as a critical event in joint disease [2-12]. In mouse models of OA, collagenolysis by matrix metalloprotease-13 [11], and aggrecanolysis by aggrecanase-2 $[5,6]$, were proven crucial for development of disease. Aggrecanolysis may

\footnotetext{
* Correspondence: staffan.larsson@med.lu.se

'Department of Orthopedics, Clinical Sciences Lund, Lund University, BMC

C12, Klinikgatan 28, SE-221 84 Lund, Sweden

Full list of author information is available at the end of the article
}

be a prerequisite for collagenolysis [13]. Molecular fragments resulting from these degradative processes appear in synovial fluid (SF), blood, and urine, and have been investigated as biomarkers for diagnosis, disease severity, onset, or progression [14-16]. The clinical diagnosis of OA relies on symptoms in combination with radiographic changes, both of which appear late in the disease process; molecular biomarkers are being tested for an earlier detection of the disease. Urinary levels of C-telopeptides of type II collagen (CTX-II) have, for example, been shown to be associated with both the presence and the progression of radiographic hip and knee OA [17]. Proteolytic aggrecan fragments are early markers of joint-matrix damage [13], and increased

\section{C) Biomed Central}


levels of proteoglycan in SF were reported in acute injury and acute inflammatory arthritis [18-21]. We showed with an assay specific for the aggrecanasegenerated ARGS neoepitope that SF levels of ARGSaggrecan are increased in human knee disease, and that measurements of this neoepitope better discriminate between health and disease than do aggrecan levels determined by methods not specific for proteolytic cleavage $[10,12]$. The hypothesis of the present study was that an association exists between SF ARGS and the development of radiographic knee OA in a cohort of individuals after meniscectomy [22-24].

\section{Materials and methods Subjects}

The study was approved by the ethics committee of the Faculty of Medicine at Lund University; informed consent was obtained from all participants. Subjects were from a cohort of 317 patients, retrospectively identified to have undergone isolated meniscectomy at Lund University Hospital in 1973, 1978, or between 1983 and 1985 [23]. The first examinations (A) were performed in 1994, 1995, and 2000, respectively, and the second examination (B), in 2004. The mean time from meniscectomy to examination A was 18 years, and the mean time between examinations A and B was 7.5 years (Table 1). As described [23], reasons for exclusion were previous knee surgery, meniscectomy in both knee compartments, osteochondritis dissecans, fracture in or adjacent to the knee, septic arthritis, osteonecrosis, any ligament injury, or radiographic signs of knee OA at the time of surgery. Of 859 identified subjects, 456 fulfilled criteria and were invited to participate at examination A; 329 responded, and 317 had radiographs taken. Here we further excluded subjects with end-stage OA (defined subsequently) of the index knee at examination A. Lack of SF or radiographic or demographic data at examination A also were reasons for exclusion. In all, 141 of the available 317 subjects were included in this study (Figure 1, Table 1). These 141 subjects were studied as one group, or stratified for absence or presence of radiographic OA (ROA) at examination A to address possible floor or ceiling effects on the SF ARGS levels created by the quantity or quality (or both) of the cartilage in the joint.

\section{Reference group}

Seventeen individuals from a previously described reference group with no known knee injury had SF at examination $\mathrm{B}$ and complete radiographic data, and were included as references (Table 1) [23].

\section{Radiographic examination}

At examination A, we obtained standing anteroposterior radiographs of the tibiofemoral (TF) joint in about 15 degrees of flexion and a skyline view of the patellofemoral (PF) joint with the knee in about 50 degrees of flexion by using a fluoroscopically positioned $\mathrm{x}$-ray beam, by using film [24]. At examination $B$, a digital $x$ ray sensor was used, and posteroanterior and lateral views of the TF joint obtained by using the fixed flexion (SynaFlexer) protocol $[25,26]$.

Joint space narrowing (JSN) and osteophytes in the TF and PF joints were graded on a 4-point scale (0 to 3, where $0=$ no evidence of JSN or osteophytes) according to, and in comparison with, images provided in the

Table 1 Characteristics of the study subjects

\begin{tabular}{|c|c|c|c|c|}
\hline & \multirow{2}{*}{$\begin{array}{l}\text { No stratification } \\
n=141(100 \%)\end{array}$} & \multicolumn{2}{|c|}{ Stratified \pm ROA at examination $A$} & \multirow{2}{*}{$\begin{array}{c}\text { References } \\
n=17(100 \%)\end{array}$} \\
\hline & & $-\mathrm{ROA}, n=63(45 \%)$ & $+\mathrm{OA}, n=78(55 \%)$ & \\
\hline Men & $116(82 \%)$ & $49(78 \%)$ & $67(86 \%)$ & $15(88 \%)$ \\
\hline Age at examination $A$, years & $51(31-73)$ & $51(32-73)$ & $52(31-73)$ & $54(37-70)$ \\
\hline BMl at examination $\mathrm{A}, \mathrm{kg} / \mathrm{m}^{2}$ & $26(18-41)$ & $26(18-35)$ & $27(21-41)$ & $26(20-31)$ \\
\hline Years between index surgery and examination A & $18(15-22)$ & $18(15-22)$ & $18(15-22)$ & $\mathrm{Na}$ \\
\hline Years between examinations $A$ and $B$ & $7.5(4.0-10.4)$ & $7.1(4.0-10.1)$ & $7.8(4.0-10.4)$ & $8.6(8.6-8.8)$ \\
\hline ROA at examination $A$ & $78(55 \%)$ & $0(0 \%)$ & $78(100 \%)$ & $1(6 \%)$ \\
\hline $\mathrm{ROA}$ at examination $\mathrm{B}$ & $106(75 \%)$ & $28(44 \%)$ & $78(100 \%)$ & $1(6 \%)$ \\
\hline End-stage $O A$ at examination $B$ & $31(22 \%)$ & $2(3.2 \%)$ & $29(37 \%)$ & $0(0)$ \\
\hline Arthroplasty or osteotomy at examination B & $2(1.4 \%)^{a}$ & $0(0)$ & $2(2.6 \%)^{a}$ & $0(0)$ \\
\hline Loss of joint space/Progression of the JSN score & $76(54 \%)$ & $22(35 \%)$ & $54(69 \%)$ & $2(12 \%)$ \\
\hline Progression of osteophytes & $66(47 \%)$ & $21(33 \%)$ & $45(58 \%)$ & $2(12 \%)$ \\
\hline Progression of ROA & $98(70 \%)$ & $35(56 \%)$ & $63(81 \%)$ & $4(24 \%)$ \\
\hline SF ARGS at examination $\mathrm{A}$, pmol ARGS/ml & $6.95(0.15-15.07)$ & $6.96(0.15-14.58)$ & $6.94(0.31-15.07)$ & $\mathrm{Nd}$ \\
\hline SF ARGS at examination $B$, pmol ARGS/ml & $\mathrm{Nd}$ & $\mathrm{Nd}$ & $\mathrm{Nd}$ & $7.19(3.63-12.72)$ \\
\hline
\end{tabular}

${ }^{a}$ One arthroplasty and one osteotomy. $\mathrm{Na}$, not applicable; $\mathrm{Nd}$, not determined. Values are expressed as numbers (\%) or mean (range). BMI, body mass index; JSN, joint space narrowing; $\mathrm{OA}$, osteoarthritis; $\mathrm{ROA}$, radiographic $\mathrm{OA}$. 


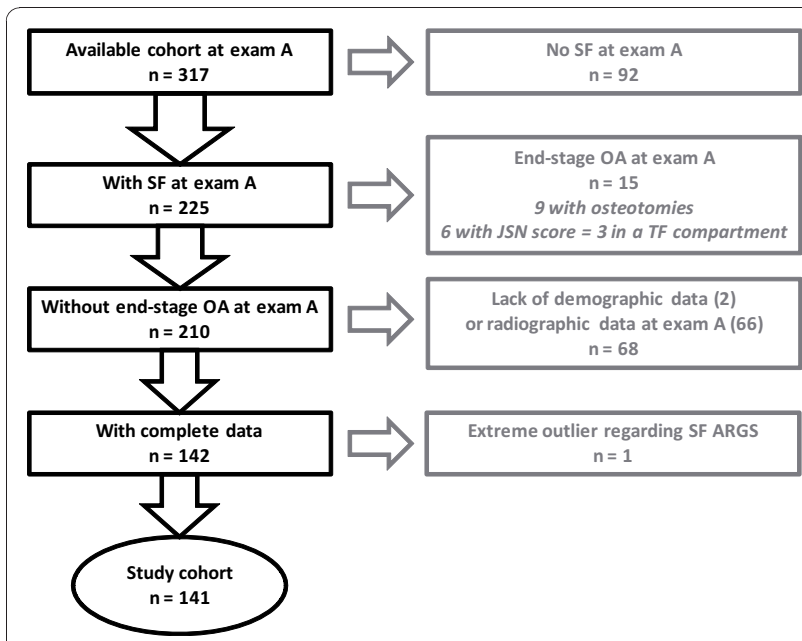

Figure 1 Flowchart of inclusion and exclusion of subjects in the study.

1995 atlas of Osteoarthritis Research Society International (OARSI) [27]. Two investigators blinded to clinical data each graded all paired radiographs with knowledge of the time sequence. Images were reread with adjudication of discrepancies between the investigators.

\section{Sum scores of JSN, osteophytes and radiographic osteoarthritis}

The sum of all JSN or osteophyte grades of an index knee were termed the JSN score or osteophyte score; the sum of all JSN and osteophyte grades in combination was termed the radiographic osteoarthritis score (ROA).

\section{Radiographic osteoarthritis score}

A knee was defined as having ROA with any of the following scores, according to the 1995 atlas of OARSI [27]:

1. JSN in any TF compartment or the PF compartment of grade 2 or higher.

2. Osteophyte score in the medial or lateral TF compartment or the PF compartment of 2 or more.

3. JSN grade 1 and osteophyte grade 1 in the same TF compartment or JSN grade 1 and osteophyte grade 1 in the PF compartment.

This cut-off approximates grade 2 TF OA on the Kellgren and Lawrence (K/L) scale [24].

\section{End-stage osteoarthritis}

A knee was considered to have end-stage ROA either (a) with JSN grade 3 in any of the TF compartments or in the PF compartment, or (b) when a subject had undergone subsequent tibial osteotomy or arthroplasty for OA.

\section{Progression of radiographic features of osteoarthritis}

We considered progression of the radiographic features of OA-loss of joint space, progression of osteophytes, or progression in either or both of those features (termed progression of ROA)-to have occurred with an increase from examination A to examination B of the JSN score, the osteophyte score, or their sum, respectively, by 1 or more in any of the TF compartments or the PF compartment. This includes both incident JSN or osteophytes at examination B and worsening of already existing changes.

\section{Materials}

Chemicals were as described [28]. Human recombinant ADAMTS-4 (a disintegrin and metalloproteinase with thrombospondin motifs; aggrecanase-1) [29], and monoclonal antibody (MAb) OA-1, specific for the N-terminal ARGS neoepitope generated by aggrecanase cleavage at the Glu-Ala bond within the aggrecan interglobular domain [30], were from GlaxoSmithKline (Collegeville, PA, USA). MAb AHP0022 against human aggrecan, described as specific for the hyaluronic acid-binding region (HABR) by the manufacturer, and as binding to both G1 and G2 of human aggrecan according to others [31], was from Invitrogen (Carlsbad, CA, USA). Chondroitinase $\mathrm{ABC}$ (EC 4.2.2.4), keratanase (EC 3.2.1.103), and keratanase II (from Bacillus sp. Ks36) were from Seikagaku (Tokyo, Japan). High-bind MA600 96-well microtiter plates (no. L11XB-1), streptavidin with Sulfo-Tag (streptavidin tagged with the reporter molecule ruthenium(II) trisbipyridyl, no. R32AD), $4 \times$ Read Buffer $\mathrm{T}$ with surfactant (no. R92TC), and the Sector Imager 6000 with software Discovery Workbench 2006 MSD_3_0_18 were from Meso Scale Discovery (MSD, Gaithersburg, MD, USA).

\section{Treatment of SF samples and standard}

Knee SF was centrifuged at 3,000 $g$ for 10 minutes at room temperature, and the supernatant was stored at $-80^{\circ} \mathrm{C}$. Twenty-five microliter aliquots of SF samples were deglycosylated for 3 hours at $37^{\circ} \mathrm{C}$ in a final volume of $32.5 \mu \mathrm{l}$ by using $0.4 \mathrm{mU}$ chondroitinase $\mathrm{ABC}$ and keratanase, and $0.02 \mathrm{mU}$ keratanase II per microliter SF in $50 \mathrm{~m} M$ Tris, $50 \mathrm{~m} M$ sodium acetate, $10 \mathrm{mM}$ EDTA, $1 \mathrm{~m} M$ AEBSF, and $10 \mathrm{~m} M$ NEM, at pH 7.3. ARGS standard was made by complete ADAMTS-4 digestion of human aggrecan, which was extracted from knee cartilage by $4 M$ guanidinium hydrochloride and purified by cesium chloride density-gradient centrifugation by using the A1D1 fraction, with a subsequent deglycosylation with chondroitinase $\mathrm{ABC}(3 \mathrm{mU} / \mu \mathrm{g})$, keratanase $(1 \mathrm{mU} / \mu \mathrm{g})$, and keratanase II $(0.1 \mathrm{mU} / \mu \mathrm{g})$, as described [28].

\section{Measurement of ARGS-aggrecan by aggrecan capture OA- 1 ARGS electrochemiluminescence (ELCL) assay}

SF levels of aggrecan fragments containing the ARGS neoepitope were analyzed by using electrochemiluminescence (ELCL) technology on the Meso Scale Discovery (MSD) platform [32-34]. 
High-bind 96-well microtiter plates were coated overnight at $4^{\circ} \mathrm{C}$ with $25 \mu \mathrm{l} /$ well of anti-human aggrecan (AHP0022) diluted to $60 \mu \mathrm{g} / \mathrm{ml}$ in PBST $(0.01 \mathrm{M}$ sodium phosphate, $0.138 \mathrm{M}$ sodium chloride, $0.0027 \mathrm{M}$ potassium chloride, $0.05 \%$ Tween 20; pH 7.4). After a wash (all washes $3 \times 400 \mu \mathrm{l}$ PBST), plates were blocked for 1 hour at $22^{\circ} \mathrm{C}$ with $150 \mu \mathrm{l} /$ well of PBST containing $1 \% \mathrm{wt} / \mathrm{vol} \mathrm{BSA}$ and $1 \% \mathrm{wt} / \mathrm{vol}$ nonfat dry milk. Plates were washed and incubated ( 2 hours, $22^{\circ} \mathrm{C}$, plate shaker) with $25 \mu \mathrm{l} /$ well of duplicates of standards ( 2.5 to 0.0073 pmol ARGS/ml) or SF (final dilutions, 1:4 to 1:26) diluted in PBST containing 1\% wt/vol BSA. After a wash, plates were incubated, as described earlier, with $25 \mu \mathrm{l} /$ well of $1 \mu \mathrm{g} / \mathrm{ml}$ biotinylated anti-ARGS (OA-1) and $1 \mu \mathrm{g} / \mathrm{ml}$ Sulfo-Tagged streptavidin in PBST containing $1 \% \mathrm{wt} / \mathrm{vol} \mathrm{BSA}$. After a final wash, $150 \mu \mathrm{l} /$ well of $4 \times$ Read Buffer diluted 1:2 in Millipore water was added, and plates were read in a Sector Imager 6000. Sample concentrations of ARGS-aggrecan were calculated from the standard curve (four-parameter logistic) by using the MSD software. A control SF was deglycosylated and plated in duplicates on each plate and used for inter- and intraassay precision. Seven SFs were spiked after deglycosylation with equimolar concentrations of standard and analyzed in the ARGS ELCL assay. To assess agreement between the ARGS ELCL assay and a published ARGS ELISA by using the same capture antibody (AHP0022) but a different detection antibody (BC-3; Abcam, Cambridge, UK) [35], 43 SF samples from a cross-sectional cohort, spanning a wide range of ARGS concentrations and diagnoses (eight with acute inflammatory arthritis, 35 with acute or chronic knee injuries) previously analyzed in the ARGS ELISA were analyzed in the ARGS ELCL assay.

\section{Western blot}

To test the specificity of the AHP0022 anti-human aggrecan antibody, $2.4 \mu \mathrm{g}$ of human aggrecan standard was deglycosylated, reduced, and separated on a $3 \%$ to $8 \%$ Tris-acetate gel, transferred, and probed with AHP0022 (1:500) or MAb OA-1 $(1: 2,000)$ by using peroxidase-conjugated horse anti-mouse IgG (CST, Danvers, MA; 1:25,000) as secondary antibody, or with antiG1 (PA1-1747; Affinity BioReagents, Golden, CO; 1:400) with peroxidise-conjugated goat anti-rabbit IgG (KPL, Gaithersburg, MD; 1:75,000), as described [28].

\section{Statistical analysis}

We used Pearson's correlation ( $r$ ) for continuous variables and Spearman's rank order correlation $\left(r_{\mathrm{S}}\right)$ when categoric variables were included. We found SF ARGS to be normally distributed, as tested with Shapiro-Wilks $(P=0.093)$. For group comparisons, we used analysis of covariance (ANCOVA); comparison of SF ARGS in male and female subjects were calculated with and without adjustments for age, BMI, and time between meniscectomy and examination A. Longitudinal associations between SF ARGS at examination A and progression of radiographic features of knee OA were assessed by using univariate and multivariate logistic regression. Odds ratios (ORs) with adjustments for age, gender, BMI, and time between examinations $A$ and B, and respective crude ORs were calculated to estimate the likelihood for progression of ROA. Progression was defined as both incident ROA and worsening of already existing ROA at examination A. We also performed analyses of radiographic progression in knees with or without ROA at examination A separately. We considered a value of $P<0.05$ to be significant. All tests were two-tailed and performed by using PASW Statistics (SPSS, Chicago, IL, USA) for Windows, version 17.0.3.

\section{Results}

\section{Technical performance of the aggrecan capture OA-1} ARGS ELCL assay

In Western blot analysis of the ADAMTS-4 digested human aggrecan used as ARGS-standard, the aggrecan antibody AHP0022 reacts with both G1-containing fragments corresponding to G1-TEGE fragments, and with the two major ARGS fragments, ARGS-SELE and ARGS-chondroitin sulfate-rich domain 1 (CS1), which both contain the G2 domain but not the G1 domain (Figure 2a). As noted by others [31], this indicates that the AHP0022, described by the manufacturer as specific for the hyaluronic acid-binding region (HABR) of aggrecan, recognizes an epitope present in both the G1 and G2 domains, which share homology [36].

Chondroitinase $\mathrm{ABC}$, keratanase, and keratanase II digestion was necessary at $0.4,0.4$, and $0.02 \mathrm{mU} / \mu \mathrm{l} \mathrm{SF}$, respectively. Lower concentrations or exclusion of any of the three enzymes resulted in decreased signal, and the addition of a hyaluronidase digestion before the described deglycosylation had no effect on the signal (not shown). With SFs diluted 1:4 or more, dilution curves of four SFs were parallel to the standard curve within the same range of ARGS concentration per well as the standard curve (Figure 2c). Table 2 details the technical performance of the ARGS ELCL assay. The mean difference between results obtained with ELISA and ELCL was $0.71 \mathrm{pmol} / \mathrm{ml}$ (Figure $2 \mathrm{~b}$ ).

\section{Patient characteristics, radiographic status, and SF levels of ARGS-aggrecan}

At a mean age of 51 years at examination A, 78 (55\%) of 141 subjects had ROA 18 years after meniscectomy. At examination B 7.5 years later, 28 additional subjects had ROA, or 106 (75\%) of 141 (Table 1). This was a considerably higher proportion than that in the reference 


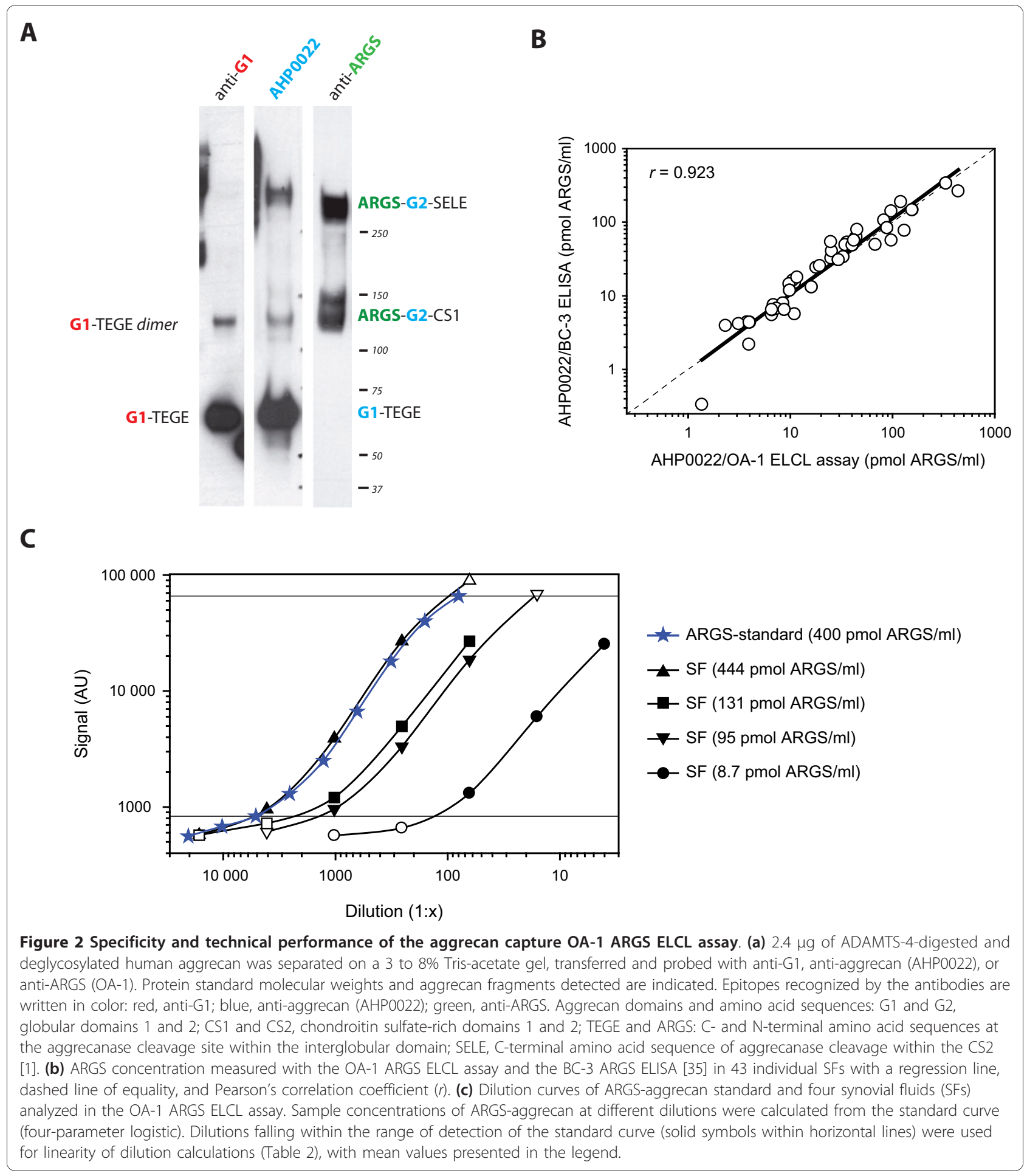

subjects, of whom one (6\%) of 17 had ROA at both examination A and at examination B 8.6 years later.

The SF ARGS levels at examination A in the 141 subjects after meniscectomy were normally distributed and ranged from 0.15 to $15.07 \mathrm{pmol} / \mathrm{ml}$, with a mean of 6.95 $\mathrm{pmol} / \mathrm{ml}$. This includes one sample of a subject who had a concentration below the level of detection that was assigned a value of $0.15 \mathrm{pmol}$ ARGS $/ \mathrm{ml}$ (that is, half the lower limit of detection). One sample of a subject had an extreme level of ARGS (31 pmol/ml), which was more than 7 times the interquartile range and was excluded. 
Table 2 Technical performance of the aggrecan capture OA-1 ARGS ELCL assay

\begin{tabular}{lc}
\hline Range of detection of standards & 0.075 to 5 pmol ARGS/ml \\
Minimum required dilution of SF & $1: 4$ \\
Lower limit of detection in SF & 0.3 pmol ARGS/ml SF \\
Parallelism (obtained/expected) of four SF samples diluted 1:4 to 1:4,000 & $100 \%(78 \% \text { to } 120 \%)^{\mathrm{a}}$ \\
Spiking recovery of equimolar spiking of seven SF samples & $116 \%(92 \% \text { to } 133 \%)^{\mathrm{b}}$ \\
Intra-assay CV, $n=14$ & $3.5 \%$ \\
Inter-assay CV, $n=9$ & $16.6 \%$
\end{tabular}

CV, coefficient of variation; ELCL, electrochemiluminescence; SF, synovial fluid. ${ }^{a} \mathrm{SFs}$ with 9, 95, 131, and $444 \mathrm{pmol}$ ARGS/ml diluted and read within the range of detection of the standard curve. ${ }^{b}$ Mean (range).

The average level and range of SF ARGS in the 68 individuals excluded from the study because of lack of demographic or radiographic data (Figure 1) did not differ from those observed in the included subjects (not shown).

No SF was available from the reference subjects at examination A; at examination B, the SF level of ARGS ranged from 3.63 to $12.72 \mathrm{pmol} / \mathrm{ml}$, with a mean of 7.19 $\mathrm{pmol} / \mathrm{ml}(P=0.78$ compared with ARGS levels in subjects after meniscectomy at examination A).

The SF ARGS concentration was higher in men compared with women, with mean (range) values of 7.34 (0.31 to 15.07$)$ and 5.14 (0.15 to 10.71) pmol ARGS/ml, respectively $(P=0.002$; Figure $3 \mathrm{a})$. The difference remained significant when adjusted for age, BMI, and time between meniscectomy and examination $\mathrm{A}(P=$ $0.005)$. BMI was higher in men $(P<0.001)$, with mean (range) values of 27.0 (21.8 to 41.4$) \mathrm{kg} / \mathrm{m}^{2}$ compared with 23.9 (17.9 to 32.0$) \mathrm{kg} / \mathrm{m}^{2}$ in women. No correlation, however, was seen between SF ARGS and BMI. No other differences, including radiographic status at examination $\mathrm{A}$, or progression thereof, were seen between men and women. SF ARGS showed no correlation with age (Figure $3 \mathrm{~b}$ ), or any of the radiographic outcome scores, alone (Figure 3c and $3 \mathrm{~d}$ ) or in combination (Figure 3e).

SF ARGS and progression of radiographic features of $O A$ Independent of stratification for ROA at examination A, a trend was seen for decreased likelihood of progression of radiographic features with increasing SF ARGS levels, with a mean odds ratio of $0.91 \mathrm{per} \mathrm{pmol} / \mathrm{ml}$ SF ARGS (Table 3). A weak but significant negative association was found with a decrease in likelihood of loss of joint space with 0.89 times per $\mathrm{pmol} / \mathrm{ml}$ increase in SF ARGS (Table 3). This association was stronger in subjects without ROA, whereas no association remained in those with ROA at examination A (Table 3).

\section{Discussion}

In this cohort of subjects with meniscectomy performed some 18 years earlier, we found SF ARGS levels no different from those in a reference group (without meniscectomy), and with no difference between subjects with ROA and subjects without. We further found that, within these relatively low and seemingly normal levels, SF ARGS and loss of joint space seem to be associated. However, contrary to our hypothesis, the association is negative: low levels of SF ARGS are associated with increased risk of loss of joint space.

We have reported, in acute inflammatory arthritis and early after injury, extremely elevated levels of SF ARGS, with a fold increase compared with healthy knee references of between 34 and 177, as measured with quantitative Western blot or ELISA, respectively $[10,12]$. We concluded that the best underlying explanation was an increased aggrecanase activity toward the interglobular domain (IGD) of aggrecan molecules that, to a large extent, were already C-terminally truncated and that the contribution from newly synthesized full-length aggrecan was minor [10,12]. The chondroitin sulfate (CS) 846 epitope has been suggested to be present mainly on recently synthesized aggrecan [37], and it was shown to be elevated two- to threefold in SF up to 20 years after knee injury compared with those uninjured [38]. In the present study, a decreased risk of joint space loss was found in subjects with higher levels of SF ARGS. A possible explanation for this could be that the higher SF ARGS levels observed here reflect a tissue-repair response involving an increased synthesis of aggrecan, in combination with aggrecanase activity. This would explain both an increase in the release of ARGS-aggrecan into the synovial fluid, and a decreased risk of loss of joint space due to an, at least in part, successful incorporation of newly synthesized aggrecan in the tissue. The fact that the negative association between SF ARGS and loss of joint space is stronger in subjects without ROA present at the examination, further strengthens the explanation that higher SF ARGS levels indeed reflect a higher synthetic activity in these subjects. Measuring only the ARGS neoepitope, we are, however, unable to ascertain the source of aggrecan fragments being degraded, which could span everything from newly synthesized aggrecan not incorporated into a functional matrix to C-terminally truncated aggrecan resident in the cartilage for a long period [39]. To better understand this balance between synthesis and degradation, our data indicate 


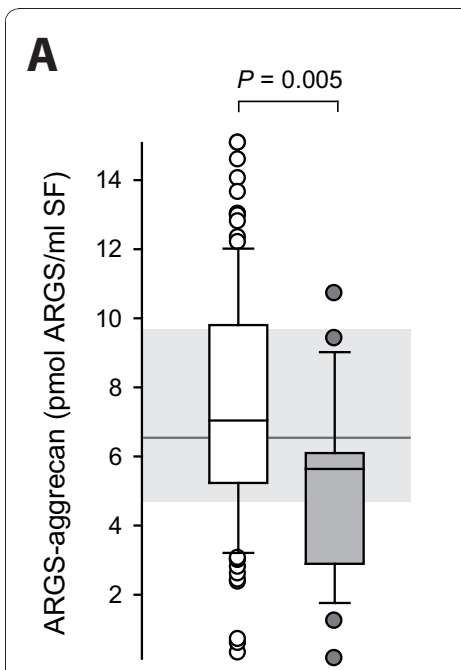

Men Women

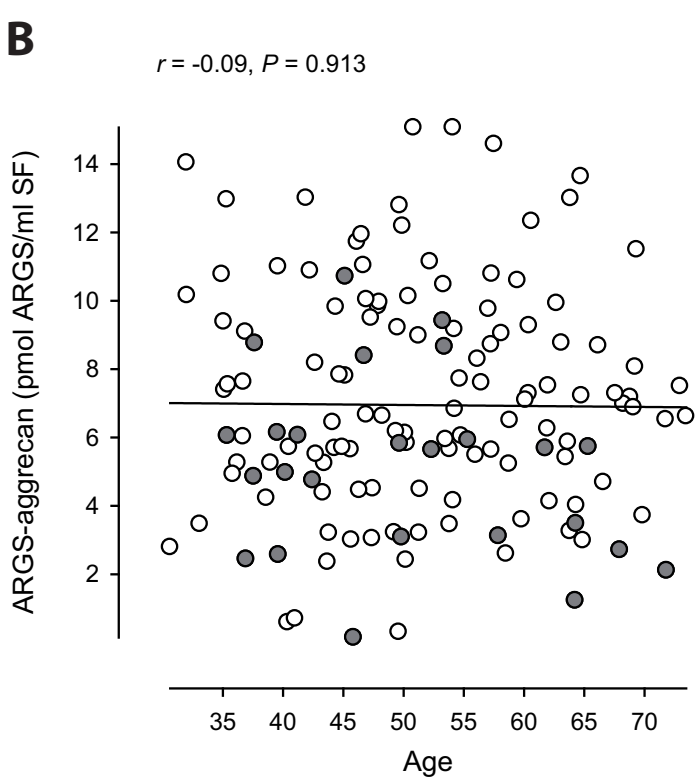

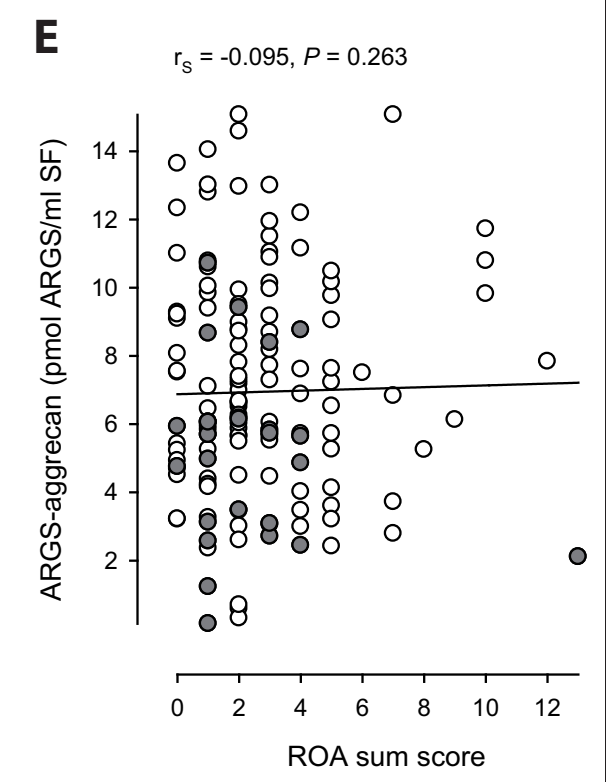
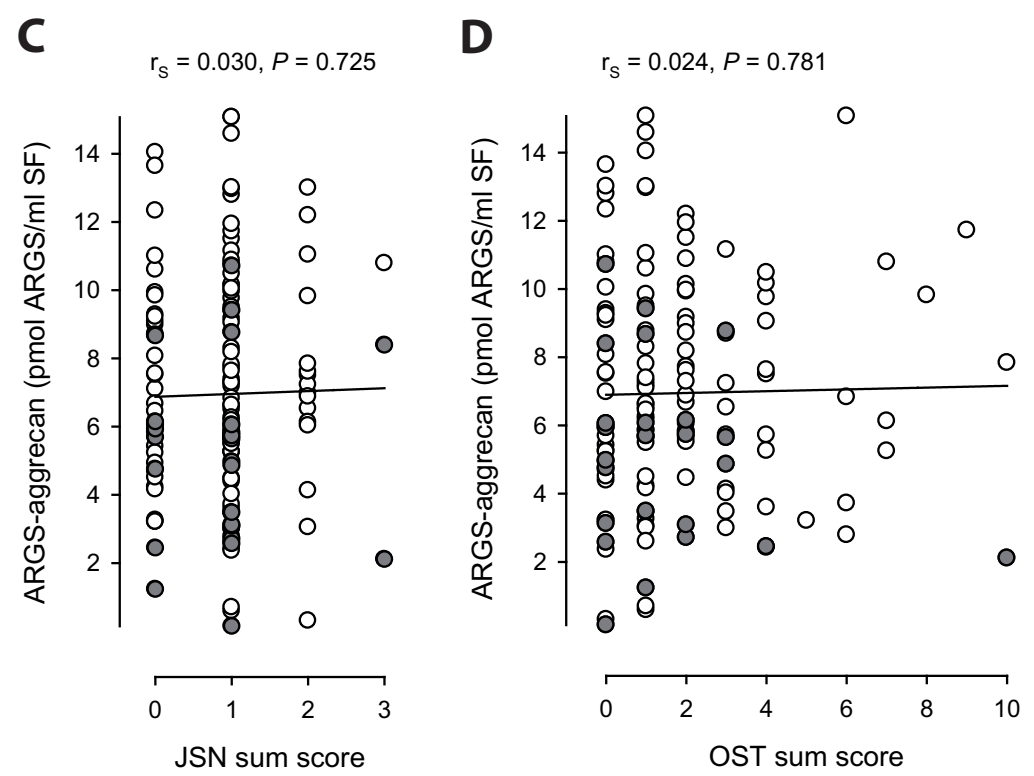

Figure 3 Distribution of SF levels of ARGS-aggrecan by gender, age, joint space narrowing (JSN) sum score, osteophyte (OST) sum score, and the sum of the JSN and OST sum scores, termed radiographic OA (ROA) sum score. Open boxes or circles represent men; grey boxes and circles represent women. (a) A box plot of SF ARGS in men $(n=116)$ and women $(n=25)$. The ends of the boxes define the $25^{\text {th }}$ and $75^{\text {th }}$ percentiles, with a line at the median, error bars defining the $10^{\text {th }}$ and $90^{\text {th }}$ percentiles and circles for individual outliers. Shaded area with line defines the $25^{\text {th }}$ and $75^{\text {th }}$ percentiles and median of the non-operated reference group $(n=17)$. Group difference was assessed by ANCOVA adjusted for age, body mass index, and time between meniscectomy and examination A. (b) Regression of SF ARGS and age with Pearson's correlation coefficient ( $r$ ). (c through e) Regression of SF ARGS and JSN, OST, and ROA sum scores with Spearman's rank order correlation coefficients $\left(r_{s}\right)$.

that the ARGS neoepitope marker may have to be used in combination with a marker of aggrecan synthesis.

Although both the quality and the quantity of the joint cartilage are suggested to influence the SF levels of biomarkers [19], we found no correlation between SF ARGS and radiographic status at the time of sampling in this dataset. Inclusion of the JSN sum score at examination A (our best approximation of joint-cartilage quantity) as an adjustment in the logistic regression model, did not essentially change the results (data not shown). Although we cannot exclude that the clearance rate of matrix molecules from the joint cavity might 
Table 3 Odds ratios (ORs) from logistic regression analyses of associations between the examination A ARGS-aggrecan levels in SF and progression of radiographic features of OA from examination A to examination B at 7.5 years

\begin{tabular}{|c|c|c|c|c|c|c|}
\hline & \multirow{2}{*}{\multicolumn{2}{|c|}{$\begin{array}{c}\text { Total sample } \\
n=141\end{array}$}} & \multicolumn{4}{|c|}{ Stratified \pm ROA at examination $A$} \\
\hline & & & \multicolumn{2}{|c|}{$-\mathrm{ROA}, n=63$} & \multicolumn{2}{|c|}{$+\mathrm{ROA}, n=78$} \\
\hline & OR & $P$ & OR & $P$ & OR & $P$ \\
\hline \multirow[t]{2}{*}{ Loss of joint space } & $0.89(0.80-0.99)$ & 0.029 & $0.77(0.63-0.94)$ & 0.012 & $0.95(0.82-1.10)$ & 0.47 \\
\hline & $0.89(0.79-0.996)$ & 0.043 & $0.77(0.62-0.95)$ & 0.016 & $0.96(0.81-1.13)$ & 0.60 \\
\hline \multirow[t]{2}{*}{ Osteophyte progression } & $0.96(0.87-1.07)$ & 0.48 & $0.90(0.76-1.07)$ & 0.23 & $1.01(0.88-1.15)$ & 0.94 \\
\hline & $0.97(0.87-1.08)$ & 0.59 & $0.92(0.76-1.11)$ & 0.38 & $0.99(0.86-1.15)$ & 0.93 \\
\hline \multirow[t]{2}{*}{ ROA progression } & $0.90(0.80-1.00)$ & 0.059 & $0.87(0.74-1.02)$ & 0.092 & $0.91(0.77-1.08)$ & 0.30 \\
\hline & $0.89(0.78-1.02)$ & 0.10 & $0.87(0.72-1.04)$ & 0.13 & $0.90(0.73-1.13)$ & 0.37 \\
\hline
\end{tabular}

Outcomes (loss of joint space, osteophyte progression, and ROA progression) are based on progression from examination A to examination B of the scores of joint space narrowing (JSN), osteophytes, and either JSN or osteophytes or both, scored according to the OARSI atlas [27]. OR and $P$ values are crude (italics) or adjusted for age, gender, BMl, and time between examinations A and B (plain text), with $95 \%$ confidence interval in parentheses.

influence the associations noted here, marker concentrations were measured long after trauma, and a steady state between markers released into the SF and markers cleared from the SF has likely occurred [40].

Multiple reports have been published on aggrecan release into SF in disease [18,19,21,38,41-43]. After knee injury, SF levels of aggrecan were initially much elevated, but with time declined toward levels seen in uninjured knees $[18,38]$. The methods used were, however, not specific for proteolytic neoepitopes, which limits the interpretation of the underlying processes causing the aggrecan release. Confirming previous results obtained by protein sequencing [44,45], we showed with Western blots that the majority of aggrecan fragments released into SF in disease are aggrecanase generated and carry the ARGS-neoepitope [12]. We further showed a strong positive association between SF ARGS and knee-joint disease including knee injury, in which greatly elevated levels early after injury declined over time, with 1-year marker levels approaching those observed in knee-healthy individuals [10]. The present study is the first to suggest that SF levels of aggrecanase-generated ARGS fragments are associated with radiographic progression of OA.

In the search for a biomarker able to predict progression of OA, sample accessibility, as well as the specificity of the marker for the joint, tissue, and molecule in question, must be taken into consideration. Joint fluid is more difficult to obtain than blood or urine, but markers in SF are more likely to reflect local joint biology compared with markers in blood or urine. The most probable source of cartilage markers in knee-joint fluid is the PF or TF joints. We therefore chose to consider radiographic progression of OA in both joints, knowing that mixed patellofemoral and tibiofemoral OA is common in this meniscectomized sample [24], and that catabolic products of the cartilage of both joints are released into the SF.

By using the BIPEDS (Burden of disease, Investigative, Prognostic of disease, Efficacy of intervention, Diagnostic of disease, and Safety of intervention) classification of OA biomarkers $[14,46]$, we showed that SF ARGS can be categorized as a diagnostic marker for disease with the capability of distinguishing a knee injured up to 1 year after injury from knees of healthy controls [10]. Here we showed that 18 years after a knee injury involving a meniscectomy, SF ARGS does not discriminate subjects with ROA from subjects without, nor can it be classified as a burden of disease marker for radiographic status. However, the association between SF ARGS and loss of joint space indicates a potential for SF ARGS as a prognostic marker for JSN.

The ELCL assay here used to measure ARGS-aggrecan is novel, based on an ELISA using a similar approach of capturing aggrecan fragments by a commercial antihuman aggrecan antibody and detecting with the BC-3 antibody directed at the ARGS neoepitope [35]. By using the same capture antibody, which is reactive against both G1- and G2-containing aggrecan fragments, together with the anti-ARGS MAb OA-1 [12,30], we found highly similar results on the same human SFs. The assay is more sensitive than the previously used keratan sulfate capture OA-1 ARGS ELISA [10,30] and is better suited for analysis of SF samples low in ARGS, such as those analyzed herein. The transition from ELISA to the ELCL format reduced the required sample volume by fourfold.

\section{Conclusions}

We found that concentrations of SF ARGS 18 years after meniscectomy were inversely associated with loss of joint space, where low levels of SF ARGS increased the risk for progression. 


\section{Abbreviations}

ADAMTS: a disintegrin and metalloproteinase with thrombospondin motifs; BIPEDS: the OA biomarker classification groups Burden of disease, Investigative, Prognostic of disease, Efficacy of intervention, Diagnostic of disease, and Safety of intervention; CS: chondroitin sulfate; CS1: CS-rich domain 1; CTX-II: C-telopeptides of type II collagen; ELCL: electrochemiluminescence; ELISA: enzyme-linked immunosorbent assay; HABR: hyaluronic acid-binding region; IGD: interglobular domain; JSN: joint space narrowing; MAb: monoclonal antibody; MSD: Meso Scale Discovery; OA: osteoarthritis; OR: odds ratio; PF: patellofemoral; ROA: radiographic OA; SF: synovial fluid; TF: tibiofemoral.

\section{Acknowledgements}

The authors thank Priya Chockalingam at Pfizer, Cambridge, MA, for sharing information on their aggrecan capture BC-3 ARGS ELISA with us, and for analyzing samples for validation. We also thank Michael Pratta and Sanjay Kumar (GlaxoSmithKline, Collegeville, PA, USA) for the kind gift of ADAMTS-4 and MAb OA-1, our laboratory engineer Maria Hansson and Gail Calvert from MSD for help in the setup of the ELCL assay, Ludvig Dahl and Björn Slaug for extraction of data, and Jan-Åke Nilsson for guidance in the statistical analysis. This study was supported by The Swedish Research Council (LSL, ME), the Swedish Rheumatism Association (AS, LSL), the Kock Foundation (AS), the King Gustaf V 80-year Birthday Fund (LSL), the Faculty of Medicine Lund University (LSL, ME), Region Skåne (LSL, ME), Magnus Bergvalls Foundation (AS), Alfred Österlunds Foundation (AS), and Swärds/ Eklunds Foundations (AS).

\section{Author details}

'Department of Orthopedics, Clinical Sciences Lund, Lund University, BMC C12, Klinikgatan 28, SE-221 84 Lund, Sweden. ${ }^{2}$ Clinical Epidemiology Research \& Training Unit, Boston University School of Medicine, 650 Albany Street, Suite X200, Boston, MA 02118, USA.

\section{Authors' contributions}

SL developed and ran the ARGS ECLC assay, carried out the statistical analysis and interpretation of data, and drafted the manuscript. ME, one of the principal investigators in the original study of meniscectomy, read and scored the radiographs together with another investigator (Ludvig Dahl) and revised the manuscript. AS contributed in the development of the ARGS ECLC assay and revised the manuscript. LSL conceived the original study of meniscectomy, collected samples, and revised the manuscript. All authors participated in the design, interpreted results, and approved the final manuscript.

\section{Competing interests}

The authors declare that they have no competing interests.

Received: 10 September 2010 Revised: 12 November 2010 Accepted: 31 December 2010 Published: 31 December 2010

\section{References}

1. Sandy JD: Proteolytic degradation of normal and osteoarthritic cartilage matrix. In Osteoarthritis.. 2 edition. Edited by: Brandt KD, Doherty M, Lohmander LS. Oxford: Oxford University Press; 2003:82-92.

2. Fosang AJ, Last K, Maciewicz RA: Aggrecan is degraded by matrix metalloproteinases in human arthritis: evidence that matrix metalloproteinase and aggrecanase activities can be independent. J Clin Invest 1996, 98:2292-2299.

3. Garnero P, Piperno M, Gineyts E, Christgau S, Delmas PD, Vignon E: Cross sectional evaluation of biochemical markers of bone, cartilage, and synovial tissue metabolism in patients with knee osteoarthritis: relations with disease activity and joint damage. Ann Rheum Dis 2001, 60:619-626.

4. Lohmander LS, Atley LM, Pietka TA, Eyre DR: The release of crosslinked peptides from type II collagen into human synovial fluid is increased soon after joint injury and in osteoarthritis. Arthritis Rheum 2003, 48:3130-3139.

5. Glasson SS, Askew R, Sheppard B, Carito B, Blanchet T, Ma HL, Flannery CR, Peluso D, Kanki K, Yang Z, Majumdar MK, Morris EA: Deletion of active ADAMTS5 prevents cartilage degradation in a murine model of osteoarthritis. Nature 2005, 434:644-648.
6. Stanton $\mathrm{H}$, Rogerson FM, East CJ, Golub SB, Lawlor KE, Meeker CT, Little CB, Last K, Farmer PJ, Campbell IK, Fourie AM, Fosang AJ: ADAMTS5 is the major aggrecanase in mouse cartilage in vivo and in vitro. Nature 2005, 434:648-652.

7. Hellio Le Graverand MP, Brandt KD, Mazzuca SA, Katz BP, Buck R, Lane KA, Pickering E, Nemirovskiy OV, Sunyer T, Welsch DJ: Association between concentrations of urinary type II collagen neoepitope (uTIINE) and joint space narrowing in patients with knee osteoarthritis. Osteoarthritis Cartilage 2006, 14:1189-1195.

8. Cibere J, Zhang H, Garnero P, Poole AR, Lobanok T, Saxne T, Kraus VB, Way A, Thorne A, Wong H, Singer J, Kopec J, Guermazi A, Peterfy C, Nicolaou S, Munk PL, Esdaile JM: Association of biomarkers with preradiographically defined and radiographically defined knee osteoarthritis in a population-based study. Arthritis Rheum 2009, 60:1372-1380.

9. Dam EB, Byrjalsen I, Karsdal MA, Qvist P, Christiansen C: Increased urinary excretion of C-telopeptides of type II collagen (CTX-II) predicts cartilage loss over 21 months by MRI. Osteoarthritis Cartilage 2009, 17:384-389.

10. Larsson S, Lohmander LS, Struglics A: Synovial fluid level of aggrecan ARGS fragments is a more sensitive marker of joint disease than glycosaminoglycan or aggrecan levels: a cross-sectional study. Arthritis Res Ther 2009, 11:R92.

11. Little CB, Barai A, Burkhardt D, Smith SM, Fosang AJ, Werb Z, Shah M, Thompson EW: Matrix metalloproteinase 13-deficient mice are resistant to osteoarthritic cartilage erosion but not chondrocyte hypertrophy or osteophyte development. Arthritis Rheum 2009, 60:3723-3733.

12. Struglics A, Larsson S, Hansson M, Lohmander LS: Western blot quantification of aggrecan fragments in human synovial fluid indicates differences in fragment patterns between joint diseases. Osteoarthritis Cartilage 2009, 17:497-506.

13. Pratta MA, Yao WQ, Decicco C, Tortorella MD, Liu RQ, Copeland RA, Magolda R, Newton RC, Trzaskos JM, Arner EC: Aggrecan protects cartilage collagen from proteolytic cleavage. J Biol Chem 2003, 278:45539-45545.

14. Bauer DC, Hunter DJ, Abramson SB, Attur M, Corr M, Felson D, Heinegård D, Jordan JM, Kepler TB, Lane NE, Saxne T, Tyree B, Kraus VB: Classification of osteoarthritis biomarkers: a proposed approach. Osteoarthritis Cartilage 2006, 14:723-727.

15. Kraus VB: Biomarkers in osteoarthritis. Curr Opin Rheumatol 2005, 17:641-646

16. Rousseau JC, Delmas PD: Biological markers in osteoarthritis. Nat Clin Pract Rheumatol 2007, 3:346-356.

17. Reijman M, Hazes JM, Bierma-Zeinstra SM, Koes BW, Christgau S, Christiansen C, Uitterlinden AG, Pols HA: A new marker for osteoarthritis: cross-sectional and longitudinal approach. Arthritis Rheum 2004, 50:2471-2478.

18. Lohmander LS, Dahlberg L, Ryd L, Heinegård D: Increased levels of proteoglycan fragments in knee joint fluid after injury. Arthritis Rheum 1989, 32:1434-1442.

19. Dahlberg L, Ryd L, Heinegård D, Lohmander LS: Proteoglycan fragments in joint fluid: influence of arthrosis and inflammation. Acta Orthop Scand 1992, 63:417-423.

20. Lohmander LS, Hoerrner LA, Dahlberg L, Roos H, Björnsson S, Lark MW: Stromelysin, tissue inhibitor of metalloproteinases and proteoglycan fragments in human knee joint fluid after injury. J Rheumatol 1993, 20:1362-1368.

21. Saxne T, Glennås A, Kvien TK, Melby K, Heinegård D: Release of cartilage macromolecules into the synovial fluid in patients with acute and prolonged phases of reactive arthritis. Arthritis Rheum 1993, 36:20-25.

22. Englund M, Roos EM, Lohmander LS: Impact of type of meniscal tear on radiographic and symptomatic knee osteoarthritis: a sixteen-year followup of meniscectomy with matched controls. Arthritis Rheum 2003, 48:2178-2187.

23. Englund M, Lohmander LS: Risk factors for symptomatic knee osteoarthritis fifteen to twenty-two years after meniscectomy. Arthritis Rheum 2004, 50:2811-2819.

24. Englund M, Lohmander LS: Patellofemoral osteoarthritis coexistent with tibiofemoral osteoarthritis in a meniscectomy population. Ann Rheum Dis 2005, 64:1721-1726.

25. Peterfy C, Li J, Zaim S, Duryea J, Lynch J, Miaux Y, Yu W, Genant HK Comparison of fixed-flexion positioning with fluoroscopic semi-flexed positioning for quantifying radiographic joint-space width in the knee: test-retest reproducibility. Skeletal Radiol 2003, 32:128-132. 
26. Kothari M, Guermazi A, von Ingersleben G, Miaux Y, Sieffert M, Block JE, Stevens R, Peterfy CG: Fixed-flexion radiography of the knee provides reproducible joint space width measurements in osteoarthritis. Eur Radiol 2004, 14:1568-1573.

27. Altman RD, Hochberg M, Murphy WA Jr, Wolfe F, Lequesne M: Atlas of individual radiographic features in osteoarthritis. Osteoarthritis Cartilage 1995, 3(suppl A):3-70.

28. Struglics A, Larsson S, Pratta MA, Kumar S, Lark MW, Lohmander LS: Human osteoarthritis synovial fluid and joint cartilage contain both aggrecanase- and matrix metalloproteinase-generated aggrecan fragments. Osteoarthritis Cartilage 2006, 14:101-113.

29. Tortorella MD, Burn TC, Pratta MA, Abbaszade I, Hollis JM, Liu R, Rosenfeld SA, Copeland RA, Decicco CP, Wynn R, Rockwell A, Yang F, Duke $J$, Solomon K, George H, Bruckner R, Nagase H, Itoh Y, Ellis DM, Ross H, Wiswall BH, Murphy K, Hillman MC Jr, Hollis GF, Newton RC, Magolda RL, Trzaskos JM, Arner EC: Purification and cloning of aggrecanase-1: a member of the ADAMTS family of proteins. Science 1999, 284:1664-1666.

30. Pratta MA, Su JL, Leesnitzer MA, Struglics A, Larsson S, Lohmander LS, Kumar S: Development and characterization of a highly specific and sensitive sandwich ELISA for detection of aggrecanase-generated aggrecan fragments. Osteoarthritis Cartilage 2006, 14:702-713.

31. Swearingen CA, Carpenter JW, Siegel R, Brittain IJ, Dotzlaf J, Durham TB, Toth JL, Laska DA, Marimuthu J, Liu C, Brown DP, Carter QL, Wiley MR, Duffin KL, Mitchell PG, Thirunavukkarasu K: Development of a novel clinical biomarker assay to detect and quantify aggrecanase-generated aggrecan fragments in human synovial fluid, serum and urine. Osteoarthritis Cartilage 2010, 18:1150-1158.

32. Deaver DR: A new non-isotopic detection system for immunoassays. Nature 1995, 377:758-760.

33. Guglielmo-Viret $V$, Thullier $P$ : Comparison of an electrochemiluminescence assay in plate format over a colorimetric ELISA, for the detection of ricin B chain (RCA-B). J Immunol Methods 2007, 328:70-78.

34. Chowdhury F, Williams A, Johnson P: Validation and comparison of two multiplex technologies, Luminex and Mesoscale Discovery, for human cytokine profiling. J Immunol Methods 2009, 340:55-64.

35. Chockalingam PS, Sun W, Rivera-Bermudez MA, Zeng W, Dufield DR, Larsson S, Lohmander LS, Flannery CR, Glasson SS, Georgiadis KE, Morris EA: Elevated aggrecanase activity in a rat model of joint injury is attenuated by an aggrecanase specific inhibitor. Osteoarthritis Cartilage.

36. Doege KJ, Sasaki M, Kimura T, Yamada Y: Complete coding sequence and deduced primary structure of the human cartilage large aggregating proteoglycan, aggrecan: human-specific repeats, and additional alternatively spliced forms. J Biol Chem 1991, 266:894-902.

37. Rizkalla G, Reiner A, Bogoch E, Poole AR: Studies of the articular cartilage proteoglycan aggrecan in health and osteoarthritis: evidence for molecular heterogeneity and extensive molecular changes in disease. $J$ Clin Invest 1992, 90:2268-2277.

38. Lohmander LS, lonescu M, Jugessur H, Poole AR: Changes in joint cartilage aggrecan after knee injury and in osteoarthritis. Arthritis Rheum 1999, 42:534-544.

39. Lohmander LS, Poole AR: Defining and validating the clinical role of molecular markers in osteoarthritis. In Osteoarthritis.. 2 edition. Edited by: Brandt KD, Doherty M, Lohmander LS. Oxford: Oxford University Press; 2003:468-477.

40. Simkin PA, Bassett JE: Cartilage matrix molecules in serum and synovial fluid. Curr Opin Rheumatol 1995, 7:346-351.

41. Lohmander LS, Dahlberg L, Eyre D, Lark M, Thonar EJ, Ryd L: Longitudinal and cross-sectional variability in markers of joint metabolism in patients with knee pain and articular cartilage abnormalities. Osteoarthritis Cartilage 1998, 6:351-361.

42. Saxne T, Heinegård D, Wollheim FA, Pettersson H: Difference in cartilage proteoglycan level in synovial fluid in early rheumatoid arthritis and reactive arthritis. Lancet 1985, 2:127-128.

43. Saxne $T$, Heinegård D, Wollheim FA: Therapeutic effects on cartilage metabolism in arthritis as measured by release of proteoglycan structures into the synovial fluid. Ann Rheum Dis 1986, 45:491-497.

44. Sandy JD, Flannery CR, Neame PJ, Lohmander LS: The structure of aggrecan fragments in human synovial fluid: evidence for the involvement in osteoarthritis of a novel proteinase which cleaves the
Glu 373-Ala 374 bond of the interglobular domain. J Clin Invest 1992, 89:1512-1516.

45. Lohmander LS, Neame PJ, Sandy JD: The structure of aggrecan fragments in human synovial fluid: evidence that aggrecanase mediates cartilage degradation in inflammatory joint disease, joint injury, and osteoarthritis. Arthritis Rheum 1993, 36:1214-1222.

46. Kraus VB, Nevitt M, Sandell LJ: Summary of the OA Biomarkers Workshop 2009 Biochemical Biomarkers: biology, validation, and clinical studies. Osteoarthritis Cartilage 2010, 18:742-745.

doi:10.1186/ar3217

Cite this article as: Larsson et al.: Association between synovial fluid levels of aggrecan ARGS fragments and radiographic progression in knee osteoarthritis. Arthritis Research \& Therapy 2010 12:R230.

\section{Submit your next manuscript to BioMed Central and take full advantage of:}

- Convenient online submission

- Thorough peer review

- No space constraints or color figure charges

- Immediate publication on acceptance

- Inclusion in PubMed, CAS, Scopus and Google Scholar

- Research which is freely available for redistribution

Submit your manuscript at www.biomedcentral.com/submit
Ciomed Central 